\title{
Evaluation and Analysis of Medical and Health Resource Allocation Efficiency in HuBei Province
}

\author{
Liu Jia ${ }^{1}$ \\ ${ }^{1}$ Capital University of Economics and Business Beijing
}

\begin{abstract}
This paper evaluates and analyzes the efficiency of medical and health resource allocation in the whole country, HuBei province and 19 regions under its jurisdiction. The factors that promote technological progress, such as system reform, management ability improvement and strong policy implementation, are the important reasons that lead to the catch-up effect of resource allocation efficiency. According to the above analysis results, this paper puts forward some suggestions to improve the practical problems.
\end{abstract}

\section{INTRODUCTION}

In December 2019, a new type of coronavirus pneumonia (COVID-19) broke out in HuBei province and spread rapidly around the world. The 19th national congress of the communist party of China (CPC) put forward the strategic plan of building a "healthy China", which provides a development route with Chinese characteristics for the reform of China's medical and health system, benefiting the whole nation, the whole cycle and all aspects. The outbreak has highlighted many shortcomings in the development of China's medical and health undertakings, such as the shortage of medical and health resources and the weak construction of rural medical and health care. Therefore, it is necessary to calculate the input and output efficiency of China's medical and health resources. Through the evaluation, we can face up to the current situation and problems in the allocation of medical and health resources, and find out the gap, the direction and method for improvement.

\section{THERE ARE GREAT DIFFERENCES IN THE} DISTRIBUTION OF MEDICAL AND HEALTH RESOURCES IN 17 CITIES AND PREFECTURES IN HUBEI PROVINCE.

The uneven distribution of medical and health resources in cities and prefectures in HuBei province is closely related to population distribution, urbanization degree and regional economic development.

\section{A. The distribution of medical and health institutions is ladder.}

From the perspective of the number of medical and health institutions, the cities and states in HuBei province are distributed in steps. According to the matching degree of medical and health institutions and population, there are several special cities. HuanGgang city's population is close to $2 / 3$ of WuHan city, and the number of medical and health institutions can catch up with WuHan city. ShiYan city also has a similar situation. XiaoGan and JingMen, on the contrary, the number of health institutions is small and the population is large. The urbanization rate of HuBei province is relatively stable.

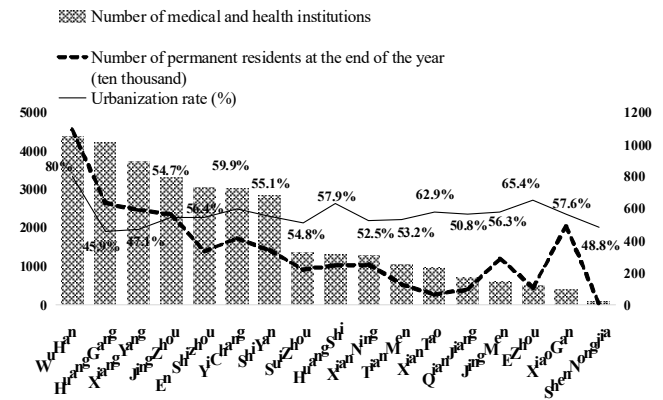

Figure 1. Population, medical institutions and urbanization of HuBei province in 2017.

B. Large differences in medical and health expenditure.

The proportion of HuBei provincial government's medical and health expenditure in the regional GDP fluctuates greatly (see Figure 2). WuHan has the largest number of medical and health institutions and the largest population, but its medical and health investment is relatively low, ranking just ahead of QianJiang and EZhou, accounting for only $1.04 \%$ of GDP. EnShi, ShenNongia and HuanGgang are far ahead of other cities in spending about $4 \%$ of GDP on health care. In the remaining 11 cities, health spending accounted for more than 2 per cent of GDP in four and more than 1 per cent in seven. In the aspect of medical and health investment, there is a ladder distribution between cities and states in HuBei province.

wdbaby0122@126.com 


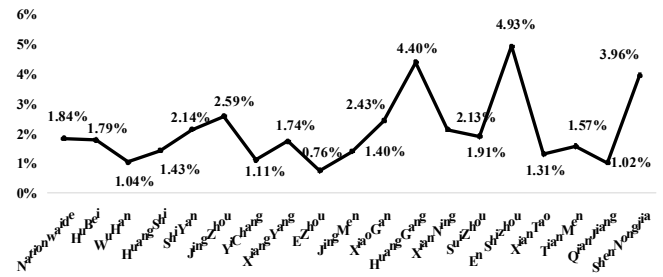

Figure 2. Proportion of medical and health expenditure in regional GDP in 2017.

\section{Uneven per capita ownership of medical and health resources.}

By comparing the number of medical and health service personnel, we can understand the actual medical service level and the real gap between different regions. According to figure 3 , the trend of the number of licensed doctors, registered nurses and beds per 1,000 population in each city and prefecture of HuBei province is basically the same. Based on the data of three indicators, the number of the areas with outstanding performance is high, including WuHan, ShiYan, YiChang, EnShi prefecture, xiangyang city and ShenNongjia forest region. The second tier consists of HuangShi city, EZhou city, JingMen city, HuanGgang city and XianNing city. The weakest areas are JingZhou (with a large population), XiaoGan, SuiZhou, XianTao, TianMen and QianJiang.

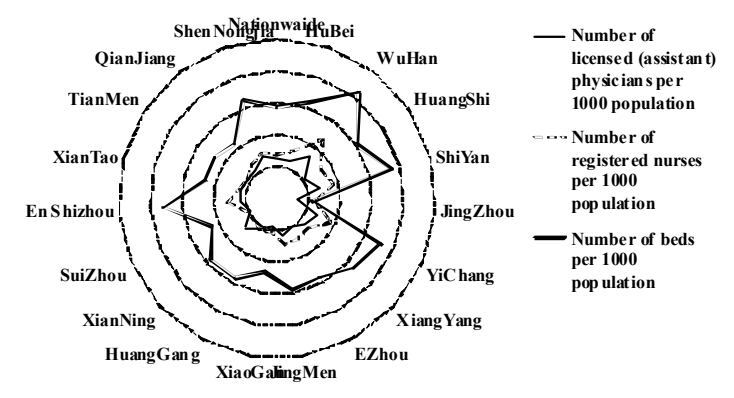

Figure 3. Medical and health conditions of HuBei province by region in 2017

\section{Empirical ANALYSIS}

\section{A. Analysis Method}

Data envelopment analysis (DEA) method is a method to evaluate the relative effectiveness under the condition of multi-input and multi-output. It is used to evaluate the efficiency between inputs and outputs of similar decision making units, compare the relative effectiveness of the efficiency between decision making units, and find the ratio of inputs and outputs close to the optimal production frontier. The models used in this paper are DEA-BC ${ }^{2}$ model for static analysis respectively.

This paper selects three indicators as the input indicators and four output indicators which showed in table 1 .

TABLE I. EVALUATION INDEX OF MEDICAL AND HEALTH RESOURCE ALLOCATION EFFICIENCY

\begin{tabular}{|c|c|}
\hline $\begin{array}{c}\text { Level } \\
\text { indicators }\end{array}$ & The secondary indicators \\
\hline
\end{tabular}

\begin{tabular}{|c|c|c|}
\hline \multirow{4}{*}{$\begin{array}{c}\text { Input } \\
\text { indicators }\end{array}$} & $X_{1}$ & $\begin{array}{c}\text { Budgetary expenditure on health care } \\
\text { and family planning (100 million } \\
\text { yuan) }\end{array}$ \\
\cline { 2 - 3 } & $X_{2}$ & $\begin{array}{c}\text { Urban per capita medical care } \\
\text { expenditure (yuan) }\end{array}$ \\
\cline { 2 - 3 } & $X_{3}$ & $\begin{array}{c}\text { Rural per capita medical care } \\
\text { expenditure (yuan) }\end{array}$ \\
\hline
\end{tabular}

\section{B. Empirical analysis results analysis}

Observation the results, we can draw the following conclusions:

- The effective allocation of medical resources in HuBei province for ShiYan, EZhou, ShenNongjia forest areas, the common point of these three areas is that the per capita access to more medical and health services, the input and output of the matching degree is relatively high.

- The areas where the pure technical efficiency is effective are WuHan, YiChang, XianNing and TianMen, but these four areas are non-effective units, which indicates that the scale efficiency still needs to be improved, and it needs to be improved from the aspects of system and management.

- Other cities in HuBei province are not effective areas for resource allocation, need to be corrected.

TABLE II. EMPIRICAL RESULTS OF MEDICAL AND HEALTH RESOURCE ALLOCATION EFFICIENCY IN 2017

\begin{tabular}{|c|c|c|c|c|c|}
\hline $\begin{array}{c}\text { Ord } \\
\text {-er }\end{array}$ & Region & $\begin{array}{c}\text { Technical } \\
\text { efficiency }\end{array}$ & $\begin{array}{c}\text { Pure technical } \\
\text { efficiency }\end{array}$ & $\begin{array}{c}\text { Scale } \\
\text { efficienc } \\
\mathbf{y}\end{array}$ & $\begin{array}{c}\text { Scale } \\
\text { Return }\end{array}$ \\
\hline $\mathbf{1}$ & Nationwaide & 1.000 & 1.000 & 1.000 & - \\
\hline $\mathbf{2}$ & HuBei & 0.717 & 1.000 & 0.717 & drs \\
\hline $\mathbf{3}$ & WuHan & 0.746 & 1.000 & 0.746 & drs \\
\hline $\mathbf{4}$ & HuangShi & 0.596 & 0.853 & 0.699 & drs \\
\hline $\mathbf{5}$ & ShiYan & 1.000 & 1.000 & 1.000 & - \\
\hline $\mathbf{6}$ & JingZhou & 0.525 & 0.557 & 0.943 & drs \\
\hline $\mathbf{7}$ & YiChang & 0.766 & 1.000 & 0.766 & drs \\
\hline $\mathbf{8}$ & XiangYang & 0.680 & 0.830 & 0.819 & drs \\
\hline $\mathbf{9}$ & EZhou & 1.000 & 1.000 & 1.000 & - \\
\hline $\mathbf{1 0}$ & JingMen & 0.643 & 0.852 & 0.755 & drs \\
\hline $\mathbf{1 1}$ & XiaoGan & 0.798 & 0.807 & 0.989 & drs \\
\hline $\mathbf{1 2}$ & HuanGgang & 0.652 & 0.834 & 0.783 & drs \\
\hline $\mathbf{1 3}$ & XianNing & 0.848 & 1.000 & 0.848 & drs \\
\hline $\mathbf{1 4}$ & SuiZhou & 0.771 & 0.773 & 0.999 & irs \\
\hline $\mathbf{1 5}$ & En Shizhou & 0.966 & 0.997 & 0.970 & drs \\
\hline $\mathbf{1 6}$ & XianTao & 0.519 & 0.712 & 0.728 & drs \\
\hline $\mathbf{1 7}$ & TianMen & 0.853 & 1.000 & 0.853 & irs \\
\hline $\mathbf{1 8}$ & QianJiang & 0.733 & 0.944 & 0.777 & drs \\
\hline $\mathbf{1 9}$ & ShenNongjia & 1.000 & 1.000 & 1.000 & - \\
\hline & mean & 0.780 & 0.903 & 0.863 & \\
\hline
\end{tabular}

C. Output shortage and input redundancy analysis.

Among the 19 evaluated regions, 4 are effective in allocating medical and health resources, and the remaining 15 are not. The pure technical efficiency of WuHan city and TianMen city is 1 , which can not be optimized by adjusting the input and output, but can only be improved by improving the scale efficiency. If the 
input does not increase, the output of $Y_{1}, Y_{2}$ and $Y_{3}$ in the output variables is generally insufficient and needs to be increased. Among them, JingZhou and QianJiang have a serious shortage of beds, and their patient carrying capacity is poor. QianJiang has a serious shortage of registered nurses. The number of township health centers in HuangShi city, XianNing city, YiChang city and EnShi prefecture still needs to be increased, and the rural medical and health resources in these areas are insufficient (see table three).

TABLE III. ADJUSTMENT VALUES OF INPUT AND OUTPUT RELAXATION VARIABLES

\begin{tabular}{|c|c|c|c|c|c|c|c|c|}
\hline $\begin{array}{c}\text { Ord } \\
\text { er }\end{array}$ & Region & $\mathbf{Y}_{\mathbf{1}}$ & $\mathbf{Y}_{2}$ & $\mathbf{Y}_{3}$ & $\mathbf{Y}_{4}$ & $\mathbf{X}_{1}$ & $\mathbf{X}_{2}$ & $\mathbf{X}_{3}$ \\
\hline $\mathbf{1}$ & HuangShi & 0.66 & & & 15 & & 718 & 343 \\
\hline $\mathbf{2}$ & JingZhou & 3.41 & 1.2 & 0.408 & & & 322 & 280 \\
\hline $\mathbf{3}$ & Yichang & 0.09 & & 0.051 & 20 & & 588 & 146 \\
\hline $\mathbf{4}$ & XiangYang & & 0.56 & 0.402 & & & & 4.1 \\
\hline $\mathbf{5}$ & JingMen & & 0.65 & 0.038 & & & 1041 & 348 \\
\hline $\mathbf{6}$ & XiaoGan & 0.8 & 0.5 & 1.521 & & & 62.8 & 27.8 \\
\hline $\mathbf{7}$ & HuanGgang & & 0.6 & 1.798 & & & 0.25 & 118 \\
\hline $\mathbf{8}$ & XianNing & 1.04 & & 1.659 & 11 & & 753 & 93.8 \\
\hline $\mathbf{9}$ & SuiZhou & 1.5 & 0.1 & 0.856 & & & 107 & \\
\hline $\mathbf{1 0}$ & En Shizhou & & 0.99 & 1.014 & 36 & 1.5 & & 448 \\
\hline $\mathbf{1 1}$ & XianTa0 & & 0.19 & 0.377 & & & 291 & 2310 \\
\hline $\mathbf{1 2}$ & QianJiang & 2.77 & 0.88 & 9.176 & & & 448 & 244.6 \\
\hline
\end{tabular}

The 12 cities in table three have both insufficient output and redundant investment, indicating that the direction of resource input is wrong and the structure of input and output also needs to be adjusted. The health input of urban and rural residents is generally redundant, and the proportion of personal medical and health expenditure is relatively high, which leaves room for further decline. Among them, the rural residents in HuanGgang city, EnShi prefecture and XianTao have more investment in health care than urban residents. JingMen, XianNing and HuangShi are the cities in which the urban residents have more medical redundancy. In addition, the government health expenditure in EnShi prefecture is redundant. Although its scale efficiency is high, its pure technical efficiency is low, resulting in a certain degree of resource waste.

\section{Efficiency and fairness in the allocation of medical and health resources.}

In 2009, China began to implement the equalization strategy of public health services. However, the unfairness of medical resource allocation in China is still quite serious, especially in rural areas. On the basis of static empirical analysis, this paper compares the urban-rural disposable income gap and comprehensive technical efficiency in 19 regions. Among them, the income gap is represented by the proportion of urban disposable income higher than rural disposable income. The results show that the rural-urban income gap is also large in the relatively effective cities with comprehensive technical efficiency, and the income gap is more than $150 \%$, such as ShenNongjia forest region and ShiYan (see Figure 4).

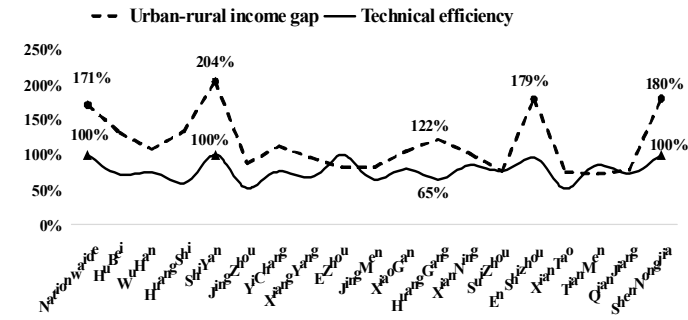

Figure 4. Urban-rural gap and efficiency of medical and health resources allocation

Under the condition that regional medical resource allocation efficiency is not different, it is impossible to separate the specific size of the allocation efficiency between urban and rural areas. However, it can be seen that while the efficiency of resource allocation is effective, the gap between urban and rural areas is too large, indicating that the allocation of medical and health resources between urban and rural areas is not fair.

\section{E. Empirical analysis conclusion}

According to the empirical analysis, we can answer the previous question.

Firstly, the total amount and per capita occupancy of medical and health resources in HuBei province are higher than the national average, but in 2017, the resources were allocated to inefficient areas, mainly because of the scale inefficiency.

Second, there is a large gap in the efficiency of medical and health resources allocation among cities and states in HuBei province. Only 3 regions are effective units, the pure technical efficiency of 3 regions is 1 , and the average comprehensive technical efficiency is only 0.78. In different periods, only YiChang, SuiZhou and ShenNongjia forest areas had Malmquist index greater than 1, which was always in a state of catching up with the frontier of resource allocation, while other cities lacked motivation to improve the efficiency of medical and health allocation.

Third, there is a large gap in the allocation of medical and health resources between urban and rural areas in some cities. On the one hand, considering the redundant investment, rural residents in HuanGgang, EnShi prefecture and XianTao have a large redundant investment and heavy medical and health burden. On the other hand, considering the insufficient output, the number of township health centers in HuangShi, XianNing, YiChang and EnShi prefecture needs to be greatly increased.

\section{Conclusions AND POLICY RECOMMENDATIONS.}

\section{A. The conclusion}

Through the empirical analysis of the efficiency of medical and health resource allocation in cities and states in HuBei province, and the derivation of the community model of medical and health level selection, I can draw the following conclusions: 
1) The regions with effective allocation of medical resources in HuBei province are ShiYan city, EZhou city and ShenNongjia forest region. The common point of these three regions is that the per capita access to medical and health services is more, and the matching degree of input and output is relatively high.

2) In most areas of HuBei province, the efficiency of resource allocation continues to decrease, while in some areas where the efficiency of medical resource allocation is relatively effective, there is a real problem of unfair resource allocation between urban and rural areas that needs to be solved urgently.

\section{B. Policy Suggestions}

1) Improve medical and health welfare per capita in urban and rural areas, especially in rural areas; We will increase the number of township health centers and their matching medical, nursing and health technicians, increase the supply of medical equipment and drugs, and effectively respond to major public health emergencies.

2) The system reform, management capacity improvement and effective policy implementation related to technological progress can improve the efficiency of resource allocation. Government departments should make full research and scientific decisions to determine the proportion of medical resources allocation according to the different effective needs of urban and rural residents, so as to improve the overall social welfare level.

3) Address regional disparities in the distribution of health resources, including between towns and cities, and between cities and villages. By improving the allocation efficiency of medical and health resources, we can ease the shortage of medical and health resources in cities and towns, encourage and support the development of medical and health undertakings and medical and health employment in rural areas, and reduce the cost of human resources in rural areas. Through the transmission force of health, improve the sense of gain and happiness of farmers, thus helping to narrow the urban-rural gap.

\section{REFERENCE}

1. Ji Jiangming, Chen xinmi. Research on the supply efficiency of rural public goods based on DEA model -- an empirical study of 15 counties (cities and districts) in zhejiang province [J]. East China economic management, 209,33 (12) : 42-48.

2. Zhao Yinyin, li liqing. Performance analysis of government healthcare investment based on dea-malmquist index model [J]. China health economy, 209,38 (12) : 26-29.

3. Li Jing, Yang fan, MAO zongfu. Equity and efficiency analysis of county-level medical and health resource allocation in HuBei province [J]. Statistics and decision-making, 2017 (13) : 114-117.

4. Xiang Fei, Wu Xiancong. Analysis on service efficiency of medical and health institutions in WuHan [J]. Medicine and society, 209,32 (05) : 10-13. 